\title{
Complex mitral valve regurgitation: surgical evaluation, approach and repair techniques
}

\author{
Chartaroon Rimsukcharoenchai ${ }^{1}$, Dimosthenis Pandis ${ }^{2}$, Ahmed El-Eshmawi ${ }^{2}$, Anelechi C. Anyanwu ${ }^{2}$, \\ David H. Adams ${ }^{2}$
}

${ }^{1}$ Division of Cardiothoracic Surgery, Chiang Mai University Hospital and School of Medicine, Chiang Mai, Thailand; ${ }^{2}$ Department of Cardiovascular Surgery, Icahn School of Medicine at Mount Sinai, NY, USA

Contributions: (I) Conception and design: All authors; (II) Administrative support: None; (III) Provision of study materials or patients: DH Adams; (IV) Collection and assembly of data: None; (V) Data analysis and interpretation: None; (VI) Manuscript writing: All authors; (VII) Final approval of manuscript: All authors.

Correspondence to: David H. Adams, MD. Department of Cardiovascular Surgery, Icahn School of Medicine at Mount Sinai, NY, USA.

Email: david.adams@mountsinai.org.

\begin{abstract}
The complexity of valve pathology in degenerative mitral valve disease has important implications in the operative approach and success of a reconstructive strategy. Originally described by Alain Carpentier's pioneering concept of pathophysiologic triad, the type and burden of mitral valve lesions are specific to the etiology of valve disease. While fibroelastic deficiency for example, is likely limited to a single segment prolapse and a ruptured chord, Barlow's valves lesions extend to involve multiple segments, clefts, chordae and the mitral annulus, requiring multiple repair techniques and an advanced level of surgical repair expertise. It is therefore important to assess disease etiology and lesion complexity during preoperative screening to plan operative approaches and anticipate operative complexity to maximize the opportunity for a successful mitral valve repair. While surgical repair techniques continue to evolve, over $95 \%$ of degenerative mitral valve lesions can be repaired successfully with targeted leaflet resection, leaflet resuspension using artificial chordae and/or native chordal transfer, and annular stabilization with a mitral annuloplasty device. With the exception of extensive annular/ leaflet calcification and some cases of significant fibrosis or inflammation which may prohibit a durable repair, most concomitant cleft, chordal, commissural and anterior leaflet pathology can be repaired successfully with a systematic lesion-specific approach, using a combination of techniques tailored to the individual valve pathology. The present article provides a stateof-the-art review of the systematic approach to complex degenerative mitral valve repair in our quaternary mitral valve reference center.
\end{abstract}

Keywords: Mitral repair, Barlow's, degenerative mitral prolapse, repair strategy

Received: 13 March 2020; Accepted: 04 June 2020; Published: 20 April 2021.

doi: 10.21037/jovs-2019-12

View this article at: http://dx.doi.org/10.21037/jovs-2019-12

\section{Introduction}

Degenerative mitral valve disease is the most prevalent valve dysfunction in the developed world (1). Mucoid degeneration often affects more than one element of the mitral apparatus, ultimately leading to incompetence. Surgical reconstruction aims to restore valve competence using a functional rather than a geometric repair approach. Although various strategies have been shown effective over the past half of the century, the wide variability in the techniques, surgical approaches and strategies have prevented attempts to standardize surgical therapy, which thus remains a case of expert opinion and individual preference.

The present review focuses on the principal tenets of our operative strategy for the surgical repair of degenerative mitral valve disease. 

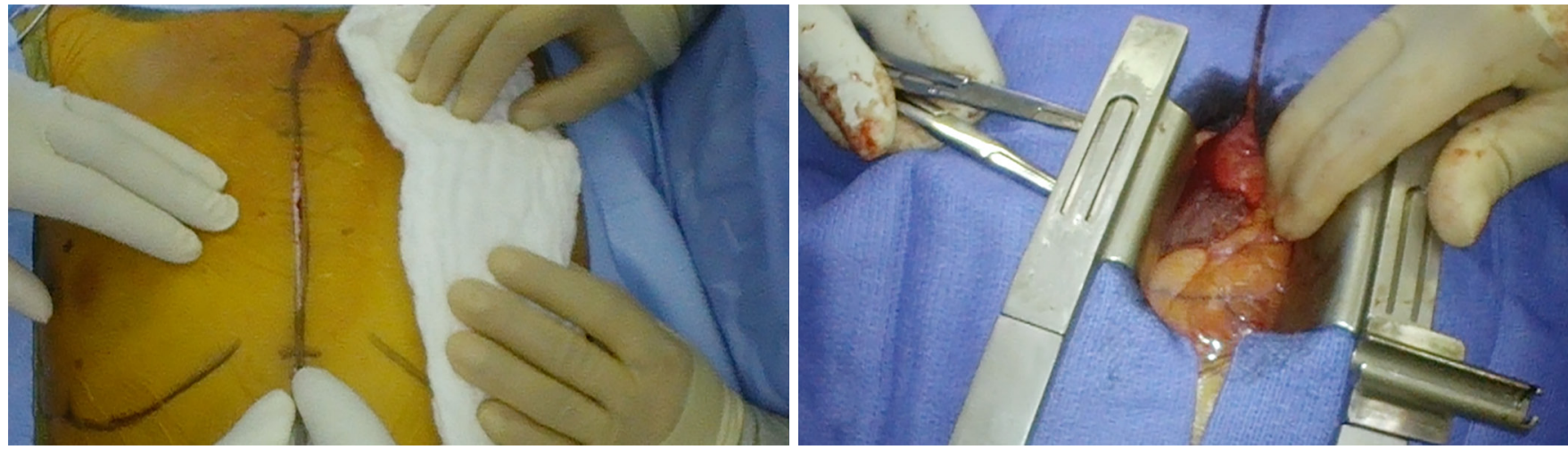

Figure 1 Example of a small access sternotomy approach for first-time degenerative mitral repair.

\section{Basic principles}

Degenerative valve disease is a process both dynamic and progressive, meaning that the central hemodynamic abnormality (valve regurgitation and incremental increase in preload) signals further valve remodeling, the emergence of new lesions and the progression of the disease (MR begets MR) until such time when ventricular compensation is surpassed and chamber dilatation heralds the onset of symptoms. As such, early repair should ensure the timely interruption of this precipitous process before the advent of symptoms, and long-term durability to effectively mitigate the risk of recurrent MR. To achieve a favorable outcome, the following principles should be applied regardless of preference in operative strategy: (I) target all areas of pathology; (II) correct excess leaflet height; (III) normalize leaflet volume; (IV) address any clefts or indentations; (V) choose an appropriate annuloplasty device and (VI) blend techniques to tailor a strategy specific to the lesions encountered (2-4).

\section{Pathophysiologic triad}

A simple yet effective method to approach valve dysfunction in the context of operative planning, devised by Alain Carpentier, is Etiology $\rightarrow$ Lesion(s) $\rightarrow$ Dysfunction (5); in other words, etiology is the cause of the valve disease (organic/primary versus functional/secondary), lesions result from the specific disease etiology and, dysfunction results from the lesions.

\section{Valve analysis}

With the recent advancements in the image quality derived from transthoracic echocardiography, including real-time 3-dimensional volume rendering, the role of transesophageal echocardiography (TEE) has been more confined to the perioperative management or, in rare cases, when TTE analysis is insufficient in clarifying the pathoanatomy of the valve (6). In our mitral reference center, we rarely request preoperative TEE when good quality TTE is available in degenerative mitral valve patients.

\section{Surgical strategy}

The attached intraoperative video demonstrates our surgical strategy for complex Barlow's cases. We perform all first-time mitral repairs using a small-access approach (Figure 1) via a $\sim 3$ in $\times 3$ in $(8 \mathrm{~cm} \times 8 \mathrm{~cm})$ sternotomy access (Figure 2), using antegrade and retrograde blood cardioplegia for myocardial protection.

\section{Operative approach}

\section{Where to start}

The following structured approach is a useful aid to translate valve analysis into repair strategy: as a first step, intraoperative assessment should aim to identify and confirm all the lesions seen on the preoperative and ontable echocardiogram; annuloplasty sutures are placed next, to improve valve exposure, followed by a secondary valve survey to determine any additional lesions not appreciated on preoperative imaging. Although control of leaflet height, volume and support are the main targets of repair, clefts, indentations and commissural pathology should also be addressed individually to ensure repair durability. In bileaflet disease, the posterior leaflet should 

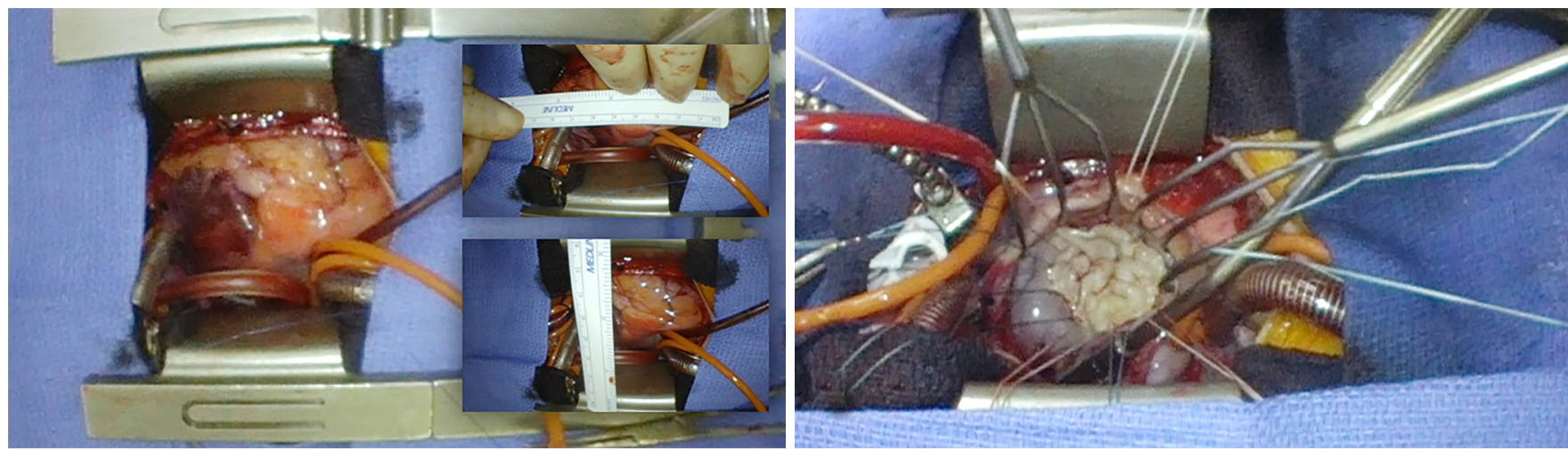

Figure 2 A small-size retractor system allows excellent exposure through a 3 in-by- 3 in $(8 \mathrm{~cm} \times 8 \mathrm{~cm})$ sternotomy access.
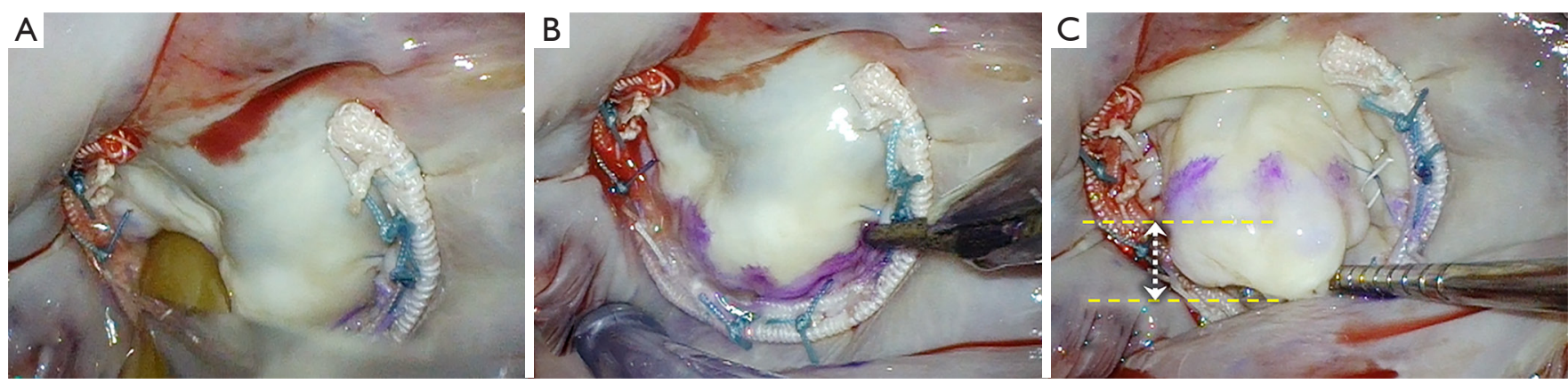

Figure 3 Intraoperative still images demonstrating (A) the saline test, (B) the ink test, and (C) assessment of the coaptation depth (white arrow line). See text for details.

be addressed first, followed by the anterior leaflet. Of note, timely identification of potential risk factors for systolic anterior motion (SAM) should always be considered during preoperative assessment, as it may impact surgical strategy. We take our time to perfect the repair and optimize the coaptation zone by pressurizing the left ventricle (saline-test) and marking the leaflet closure line (ink-test) to ensure that at least $8 \mathrm{~mm}$ of coaptation surface depth is below the ink mark line (Figure 3).

\section{Leaflet resection: tailored approach}

Resection strategies aim to correct excess height and volume in target areas of pathology while avoiding undue tension of the reconstructed leaflet. As a rule, we increasingly favor a more "respect" approach to leaflet tissue, with targeted resection limited to the segment with the most pathologic stigmata, followed by a tension-free leaflet reconstruction. Adjacent clefts and indentations can be partially closed via free-edge remodeling.

\section{Leaflet height/volume management}

\section{Triangular resection}

Triangular resection is a versatile technique that can adapt to correct leaflet height and/or volume by simply changing the height and/or angle of the two triangle's sides with the triangle's base oriented toward the leaflet's free margin (Figure 4):

(I) Small triangle: symmetric (Figure 5);

(II) Larger triangle: symmetric (Figure 6);

(III) Larger triangle: asymmetric (Figure 7).

\section{Quadrangular resection}

Quadrangular resection is an effective strategy to reduce both leaflet volume and height when excess tissue involves all segments of the posterior leaflet or both leaflets (as seen with Barlow's and forme fruste). Originally devised by Alain Carpentier, quadrangular resection takes advantage of all the available leaflet 

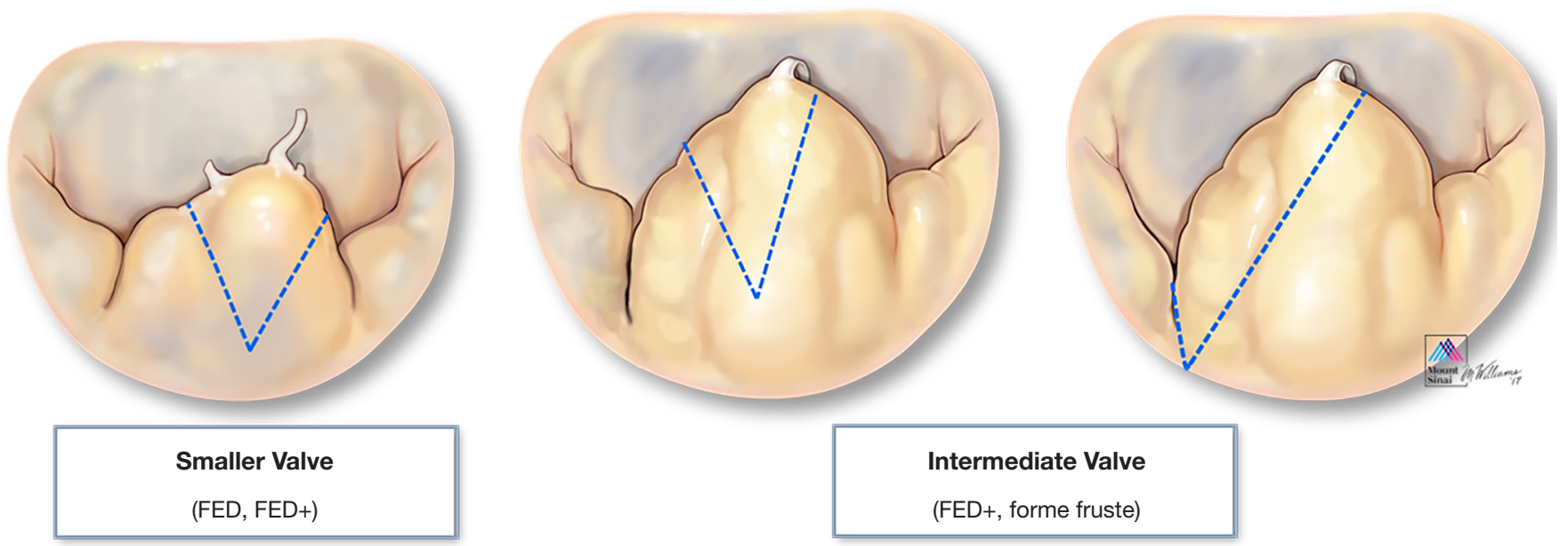

Figure 4 Graphic illustration of different types of triangular resection. FED, forme fruste.
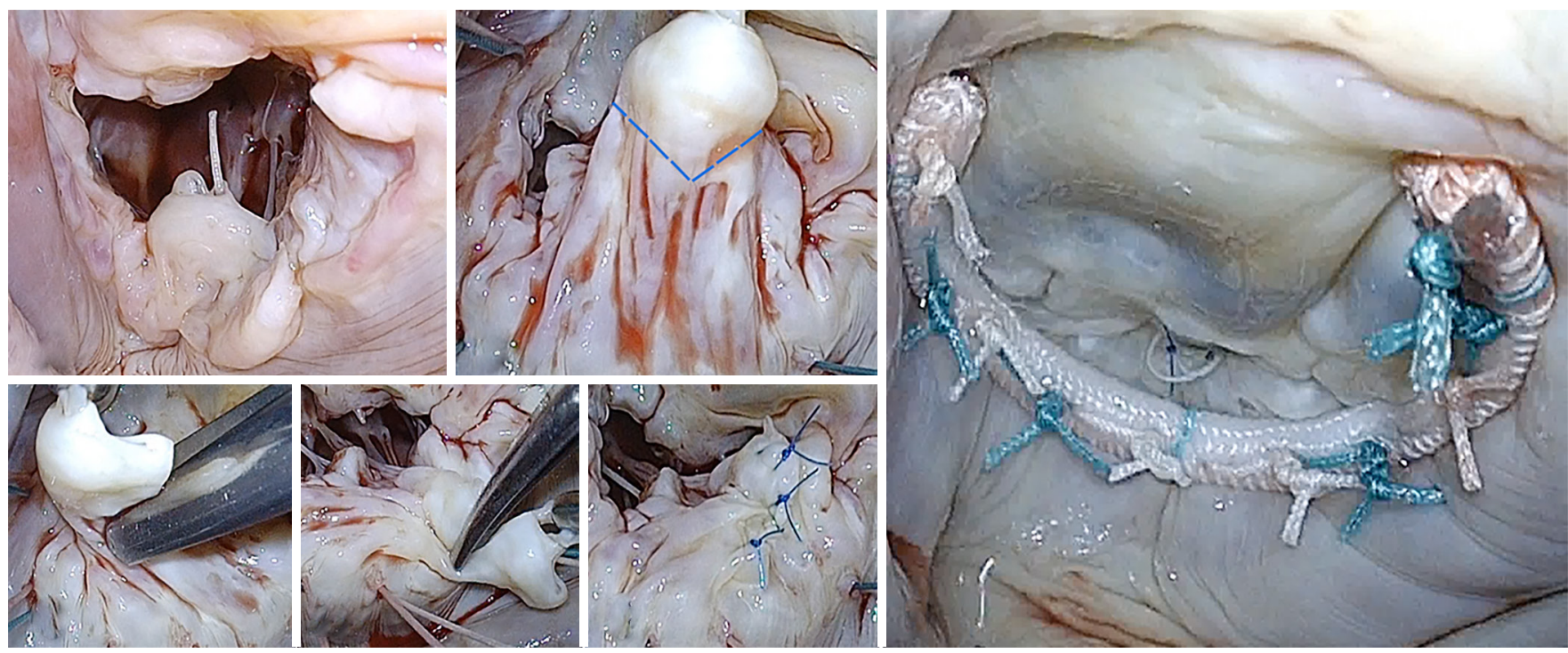

Figure 5 Targeted triangular resection in a patient with fibroelastic deficiency and ruptured chordae leading to a flail P2 segment. Note the otherwise normal valve anatomy. The repair was reinforced with an artificial chord implantation at the free margin of the reconstructed segment and a true-sized $28 \mathrm{~mm}$ annuloplasty band.

surface area by removing part of the leaflet (from free edge to annular hinge), and detaching either one or both remaining segments on either side of the resection along their annular insertion to appropriate/advance the resection margins and reconstruct the leaflet (Figure 8 ). Alternatively, when the available leaflet tissue is not sufficient (hypoplastic, calcification, endocarditis etc.) annular plication may be a useful strategy to achieve a tension-free reconstruction by reducing the resection gap (reducing the annular perimeter). In our mitral reference center, we have increasingly adopted a more conservative approach to leaflet resection, favoring the use of smaller, targeted asymmetric triangles combined with sliding leaflet plasty which allows for effective control of leaflet size while also maintaining optimal coaptation depth following reconstruction (Video 1). 

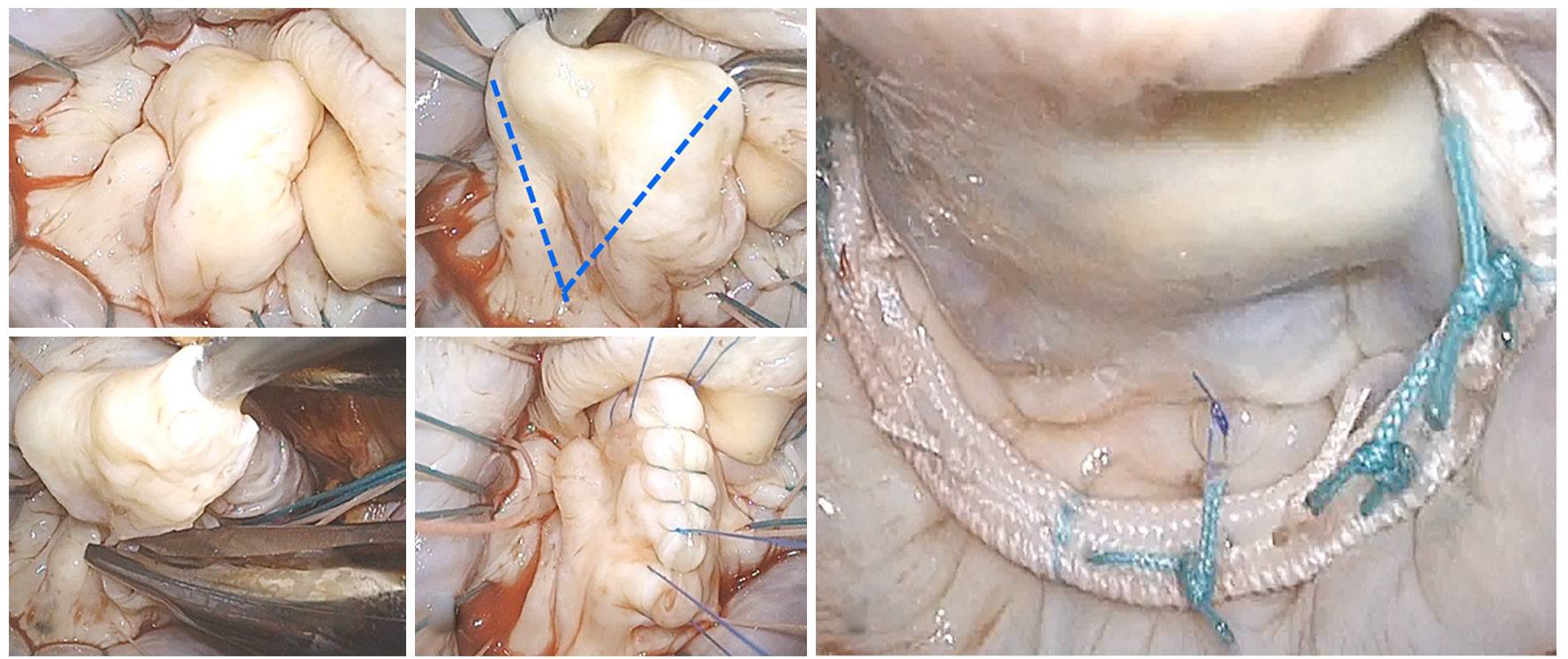

Figure 6 A larger symmetric triangular resection is used in a case where both height and volume excess are appreciated at the P2 segment of this example with FED+ pathology.
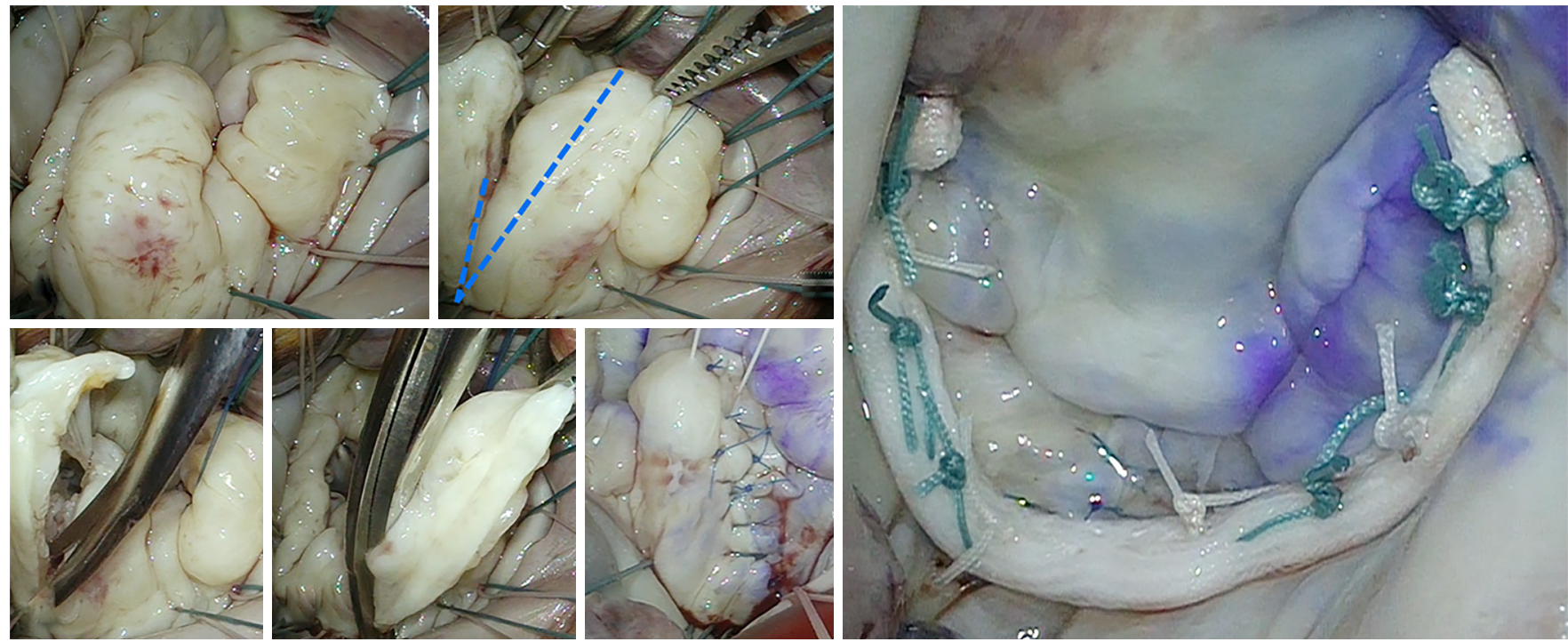

Figure 7 An example of a multi-segment leaflet prolapse and a deep cleft, in a case of forme fruste Barlow's addressed with an asymmetric triangular resection including the cleft as the lateral side of the triangle.

\section{Chordal management}

Leaflet support is an integral element of every reconstructive strategy and requires careful assessment during valve analysis. The main purpose of chordal management is twofold: (I) to support poor-quality primary chordae (i.e.,
FED) and (II) ventricular leaflet displacement to prevent SAM. Native chordae integrity and height (or length) can help guide the margins of leaflet resection (often defined as the point on the leaflet's free edge with healthy chordae just adjacent to the affected segment), and to identify the physiologic plane of leaflet closure. 

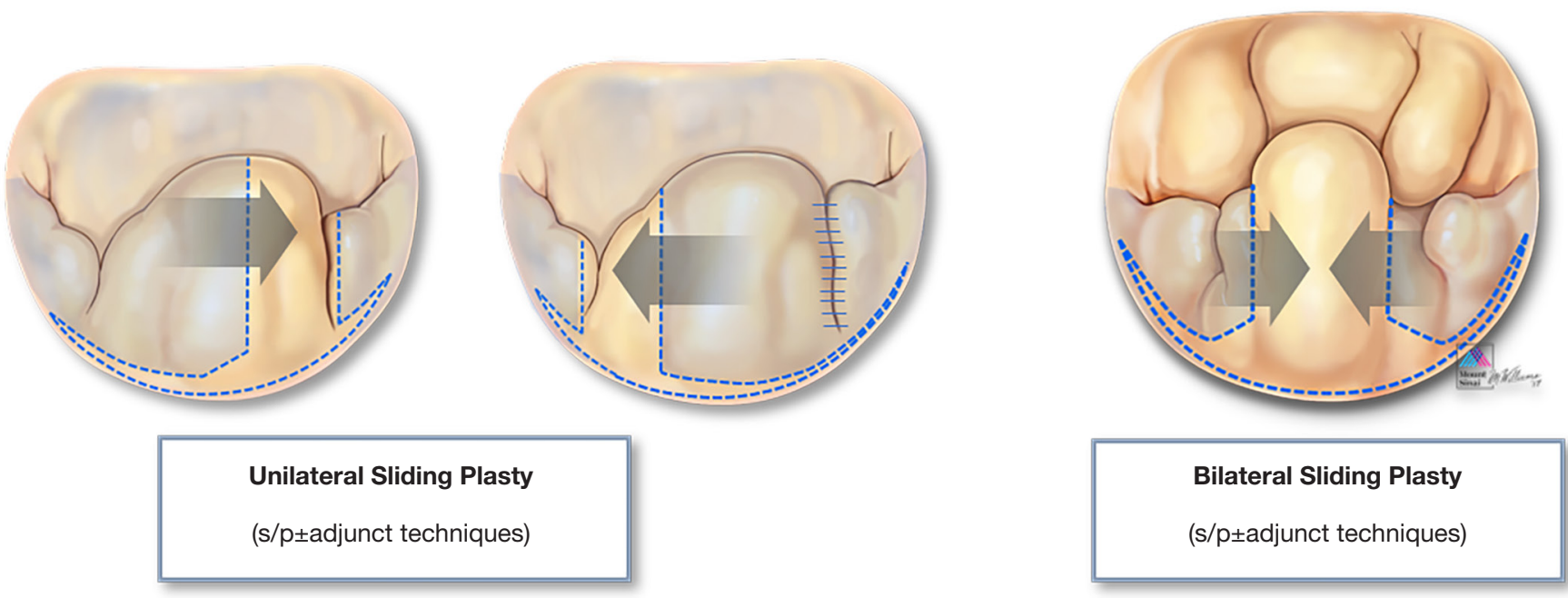

Figure 8 Graphic illustration of various types of quadrangular resection and leaflet sliding plasty techniques (see text for details). s/p, sliding plasty.

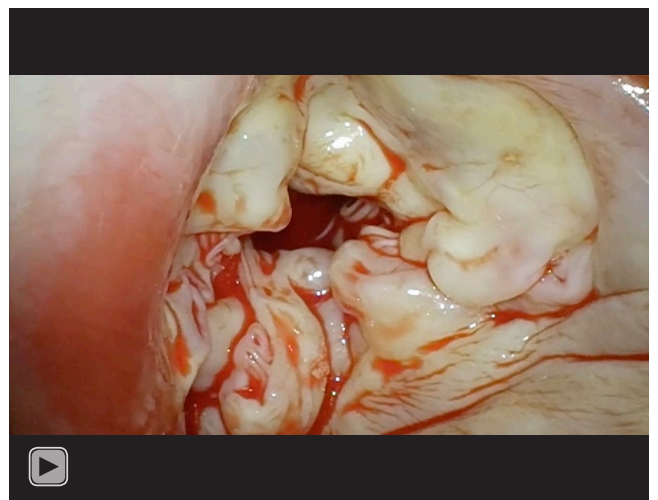

Video 1 Surgical strategy for complex Barlow's cases.

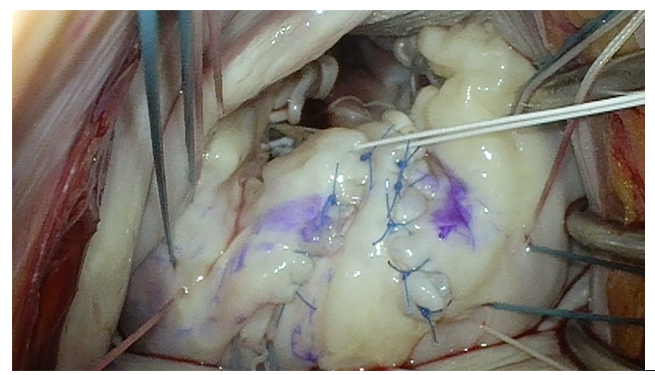

\section{Chordal replacement}

Artificial chordae from interweaved fibers of expanded polytetrafluoroethylene (ePTFE) can offer new support, reinforce the existing native chordal support and/or displace the target segment further in the ventricle to ensure a deep posterior coaptation depth during valve closure. This method may be used as an adjunct (Figure 9) or as the primary repair strategy in non-resection repair techniques (Figure 10).

\section{Chordal transfer}

It is mostly reserved for anterior leaflet prolapse, whereby a healthy secondary chord is divided at its leaflet insertion

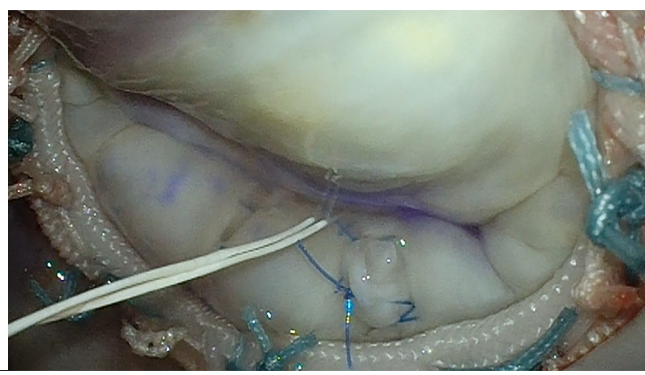

Figure 9 Support of the reconstructed segment is reinforced with an ePTFE neochord. 

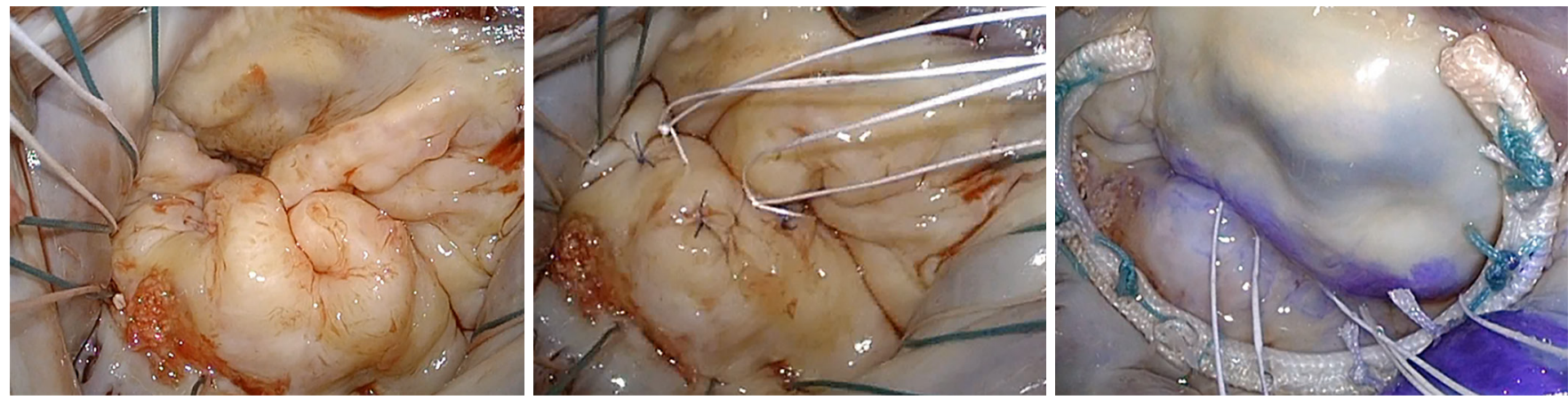

Figure 10 Ventricular displacement of the prolapsing posterior leaflet is a feasible alternative when extensive annular and leaflet calcification prevents a durable targeted repair.
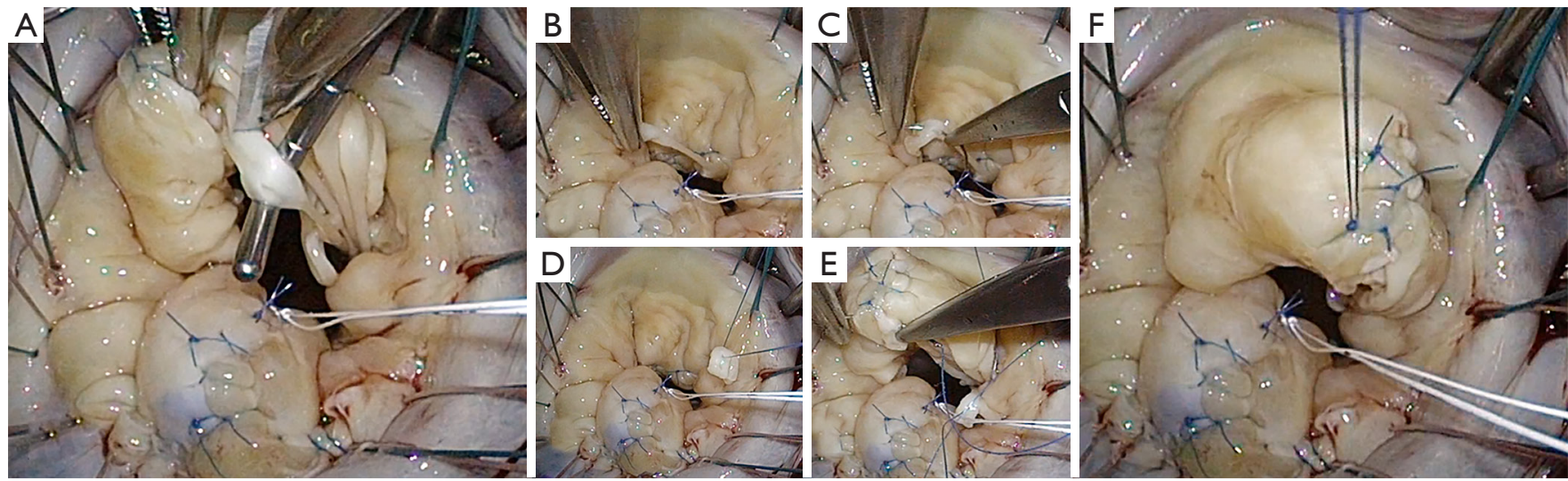

Figure 11 A healthy secondary chord is identified and divided from its leaflet insertion point (A,B). The native chord is then mobilized to the free edge of the desired segment and sutured using 4-0 Prolene to re-establish support of the target area (C-F).

point and re-inserted (transferred) to the free margin of the segment being repaired. It may be reinforced with an additional artificial chord, implanted at the same papillary tip with the transferred native chord (Figure 11).

\section{Chordal division}

A lesion often encountered in complex advanced Barlow's disease is the fibrotic thickening and/or fusion of one or more chordae (Figure 12), leading to retraction and subsequent tethering of the attached segment(s). It is also seen in hypoplastic leaflet segments, or due to calcific degeneration, chronic atrial fibrillation or an infectious process. In such cases, division of the restrictive chordae is necessary where possible, and mobilization of the leaflet body to assess the extent in which the undermined segment can contribute to the coaptation or whether it should be included in the primary repair strategy (Figure
13). Chordal division is also part of sliding leaflet plasty whereby secondary and tertiary chordae from the detached leaflet are divided to avoid tethering and undue tension after the segment is mobilized medially during leaflet advancement.

\section{Clefts and indentations}

As leaflet volume expands normal clefts and indentations may appear to increase in depth and/or prolapse or become restricted depending on the associated lesions and involvement of their chordae support. An effective method to address these lesions is based on (I) their proximity to the primary target of repair (i.e., the prolapsed leaflet) and (II) the availability of pliable leaflet tissue. When deep clefts are found at either side of the main prolapsing segment, they can be either sutured creating a single segment to address 

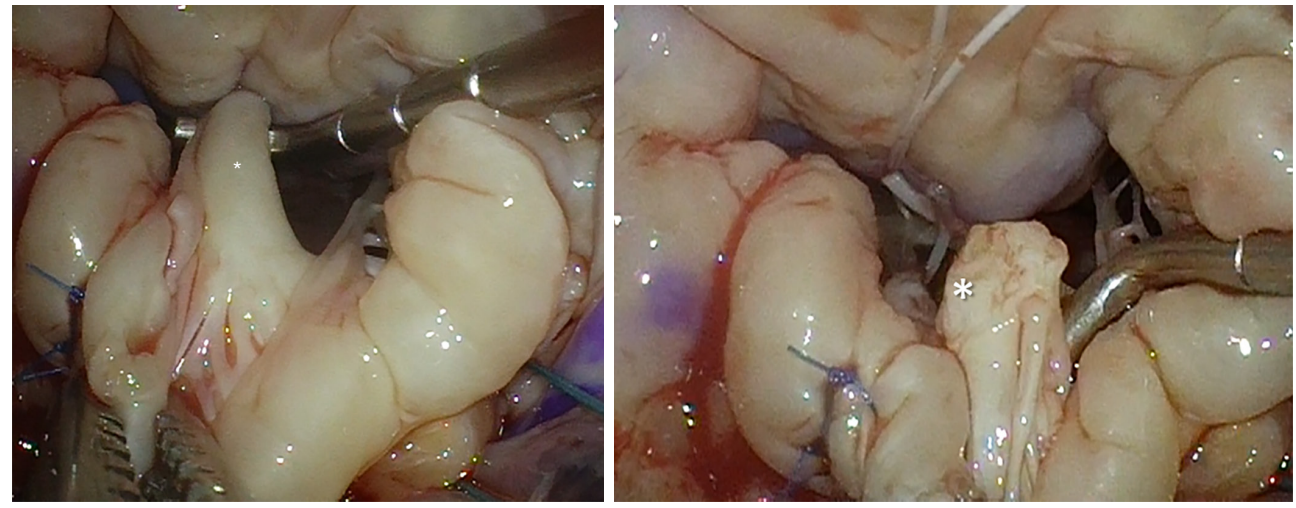

Figure 12 Fibrotic fusion $\left(^{*}\right)$ of strut chord to the posterior leaflet in a case of Barlow's deformity and chronic atrial fibrillation.
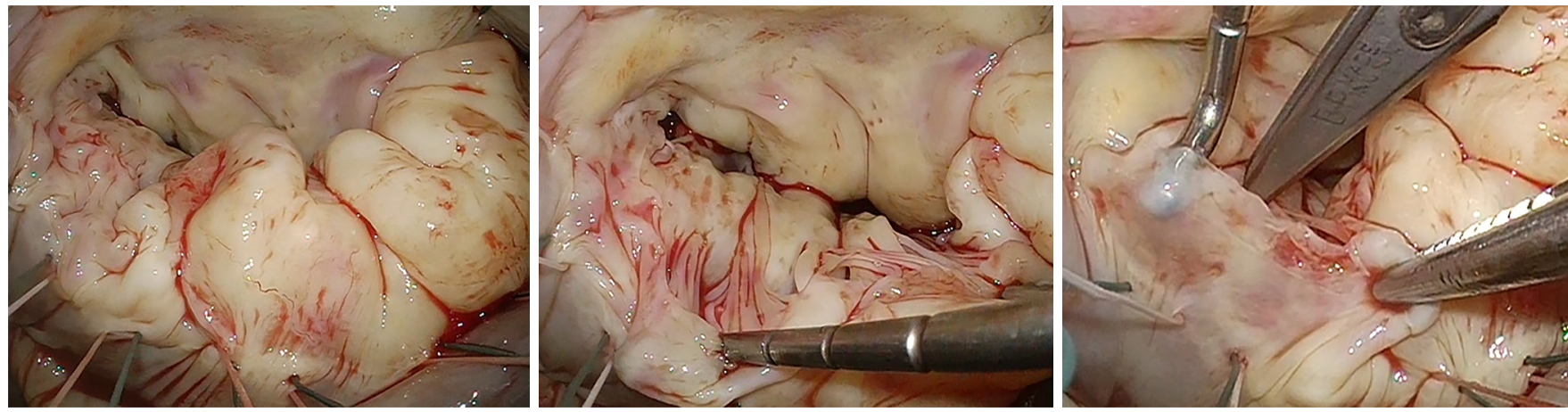

Figure 13 A hypoplastic P1 segment with significant tethering from subvalvular calcification encroaching the basal ventricular wall and adjacent support structures (papillary muscle and chordae). Sharp division of the restrictive chordae can mobilize the available leaflet tissue surface area.

with a resection technique, or become one of the resection margin, the other taken at the tallest point of the leaflet's body, followed by reconstruction. When a deep cleft is far from the planned repair, it can be closed primarily provided suturing doesn't create tension or restriction. If primary closure is likely to result in leaflet tension, or when regional tissue availability is limited (infective lesions, hypoplastic segment, calcification or chordal tethering), a first step should be to attempt mobilizing the available free margin by dividing restrictive chordae to maximize the amount of leaflet surface available for apposition. The next step is to evaluate whether the regional tissue deficit may be corrected by detaching and advancing the adjacent voluminous leaflet segment(s) toward the tissue deficit to recreate enough leaflet surface for deep coaptation with the opposite leaflet without tension (Figures 7,8).

\section{Calcification: the Mount Sinai MAC Algorithm}

A common lesion seen with advanced Barlow's deformity and further precipitated by age and systemic disease, calcification may be limited to the mitral annulus (MAC), or extend to encroach the leaflet, chordae, papillary muscle tips and basal ventricular myocardium. Limited calcification may still allow annular suture placement and a successful repair, with some experts opting to remove the fibrous envelope en-block, with the alternative of a conservative non-resection strategy (leaflet resuspension with placement of artificial chordae) or palliative maneuvers such as edge-to-edge repair. In extensive horseshoe $\left(<270^{\circ}\right)$ or circumferential MAC $\left(\geq 270^{\circ}\right)$ placement of annular sutures may be hindered, prohibiting the use of annuloplasty devices, while restricting the feasibility of secure deployment of a replacement valve prosthesis. We 


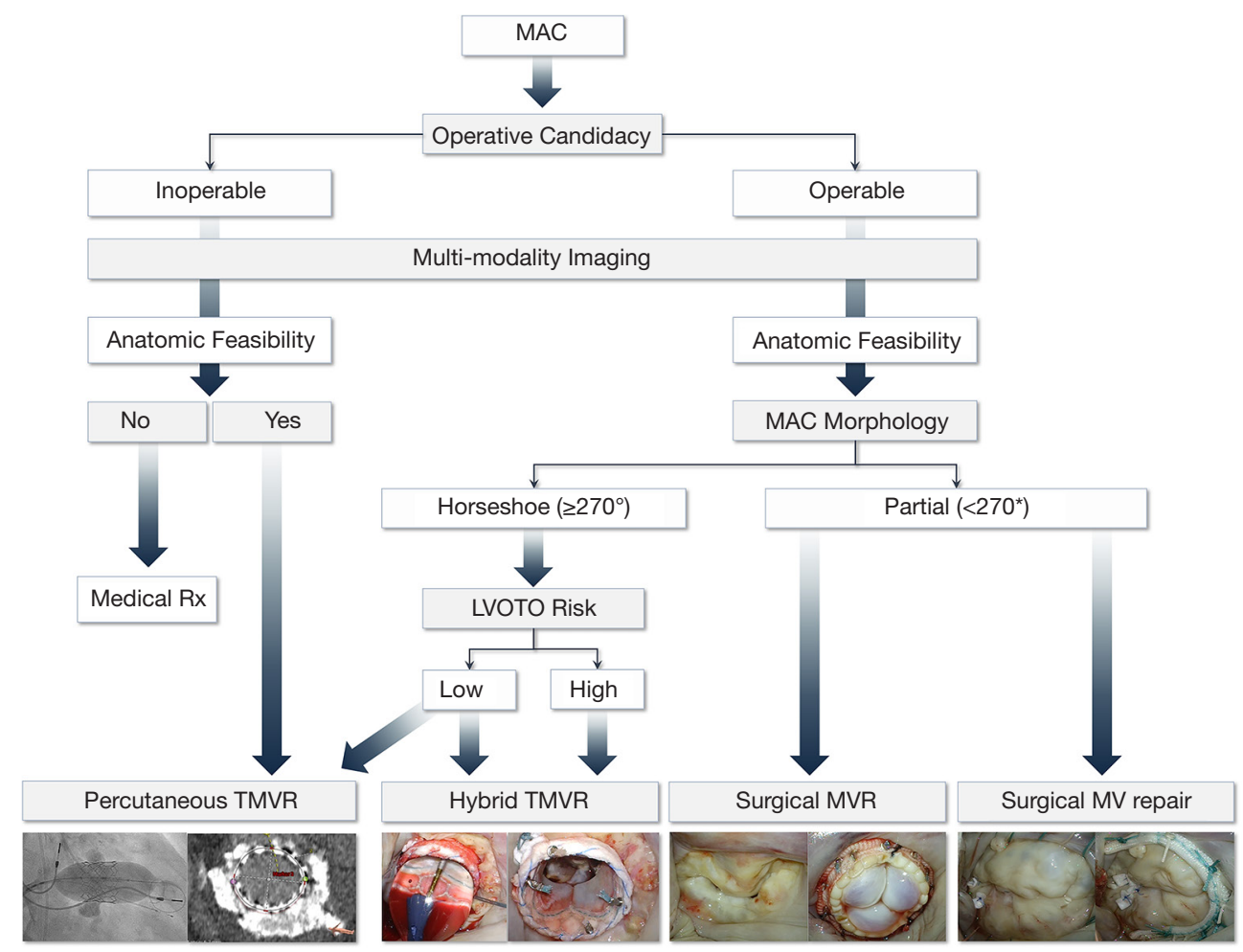

Figure 14 Following evaluation of the operative risk by the Heart Team, the anatomic characteristics of the calcified structures are evaluated to ascertain the feasibility of surgical intervention (7). LVOTO, left ventricular outflow tract obstruction; MAC, mitral annular calcification; MVR, mitral valve replacement; TMVR, transcatheter mitral valve replacement.

have developed an algorithm to identify the optimal surgical strategy using advanced imaging and multidisciplinary patient screening to tailor a patient-specific operative approach (7) (Figure 14).

\section{Annuloplasty strategies: rings and bands}

Beyond functional reconstruction of the mitral valve leaflets, annuloplasty strategies extend repair durability by restoring the physiologic functional support of the mitral annulus which in turn preserves optimal leaflet coaptation, minimizes stress on the posterior mitral leaflet and posterobasal ventricular myocardium during the cardiac cycle, reinforces the repair suture line and prevents future annular dilatation in myxomatous valve degeneration. While annuloplasty rings and bands can both achieve remodeling of the mitral annulus shape and function, with varying degrees of reduction in the septolateral (SL) and intertrigonal (IT) dimensions, we have increasingly adopted a more conservative strategy with an almost exclusive use of flexible bands in degenerative mitral valve prolapse. The use of a complete semi-flexible ring is reserved for cases where a more pronounced remodeling is desired by fixing the annulus in the systolic position, maintaining the SL/IT ratio of 3:4 throughout the cardiac cycle.

\section{$S A M$}

It is mandatory to identify risk factors for SAM in the preoperative TTE/TEE (i.e., small aorto-mitral angle, basal septal hypertrophy or reduced distance between the point coaptation and basal septum from any etiology). If present, more leaflet resection and/or chordal displacement and an oversized band annuloplasty is recommended $(8,9)$.

\section{Final assessment of repair}

Regardless of the preferred operative approach, long term durability of surgical mitral repair and the associated incremental event-free survival depends primarily on leaving the operating room with no residual or trace MR. The 


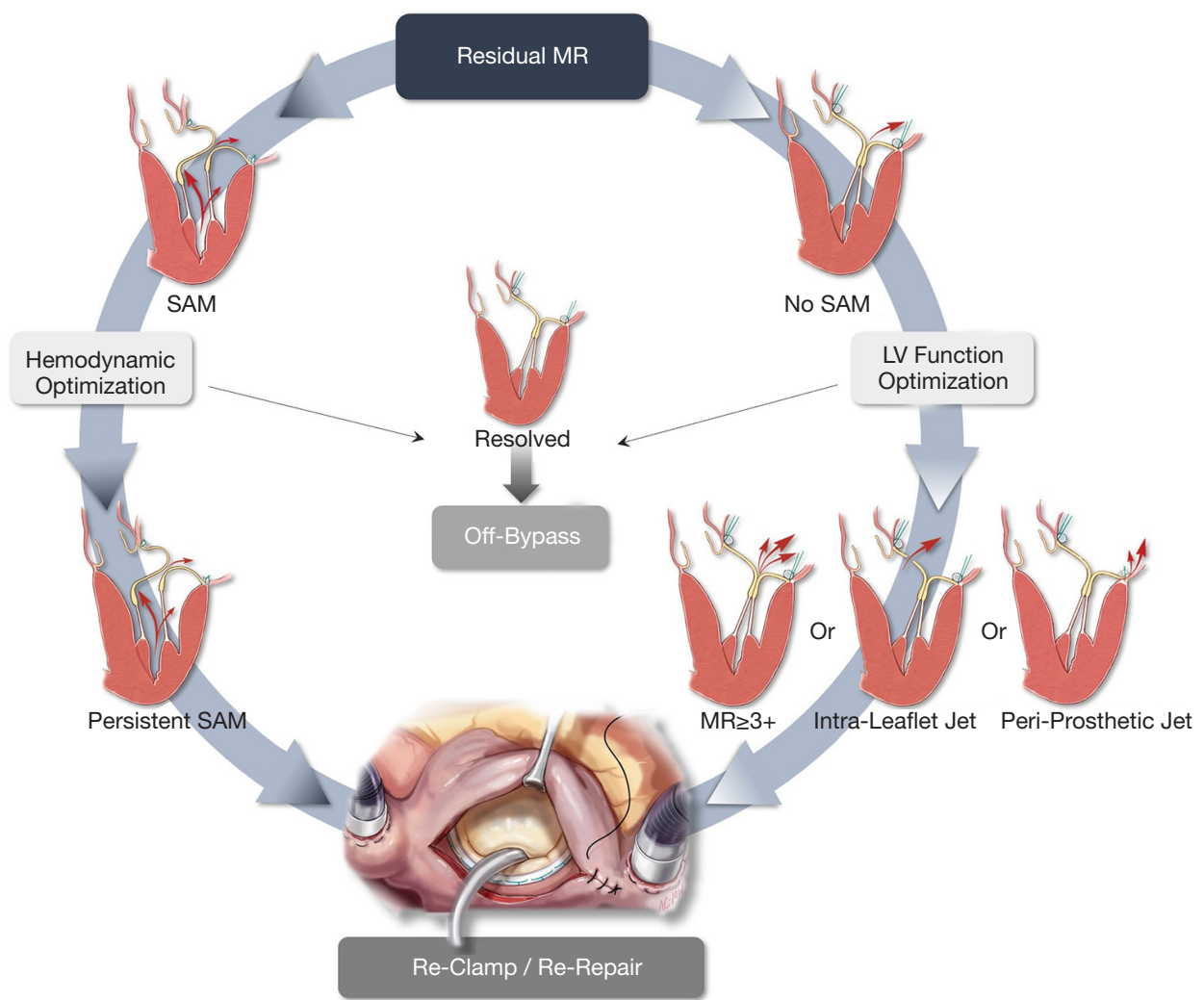

Figure 15 The Mount Sinai Algorithm for addressing residual MR following surgical mitral valve reconstruction (9).

projected durability of the repair result is thus contingent on tension-free, well-supported leaflets, with a posteriorly displaced apposition line, achieved by sufficient coaptation depth of at $\sim 10 \mathrm{~mm}$ and reinforced by a true-sized annuloplasty ring or band which affords an unrestricted inflow area and an unobstructed $\mathrm{LV}$ outflow. Intraoperative TEE can help assess the final result and identify any such areas of concern. The decision to revise the repair should be carefully assessed, balancing the competing risks between the patient's baseline morbidities and the long-term impact of the observed hemodynamic profile (i.e., SAM, residual MR). In our mitral reference center we have adopted a zero-tolerance approach for residual MR and developed a decision-making algorithm to stratify the use of a second bypass run based on our experience in employing a revision strategy to effectively mitigate significant $M R$ in select patients (9) (Figure 15).

\section{Take home points}

Complex degenerative mitral valve prolapse can be repaired successfully with a methodical assessment of the mechanisms of dysfunction, followed by a lesion-specific approach tailored to the thoughtful control of the excess height, volume and leaflet support, by blending techniques to address all areas of pathology and restore valve competence with long term durability.

\section{Acknowledgments}

Funding: The present work was funded in its entirety by the Department of Cardiovascular Surgery at The Icahn School of Medicine at Mount Sinai, NY, USA.

\section{Footnote}

Provenance and Peer Review: This article was commissioned by the Guest Editors (Filip Casselman and Johan van der Merwe) for the series "Aortic and Mitral Valve Innovative Surgery" published in fournal of Visualized Surgery. This article has undergone external peer review.

Conflicts of Interest: All authors have completed the ICMJE uniform disclosure form (available at https:// 
jovs.amegroups.com/article/view/10.21037/jovs-201912/coif). The series "Aortic and Mitral Valve Innovative Surgery" was commissioned by the editorial office without any funding or sponsorship. DHA serves as the National Co-PI for the following US Pivotal Trials: Medtronic CoreValve US Pivotal Trial, NeoChord System US Pivotal Trial, Medtronic Apollo US Pivotal Trial, Triluminate II US Pivotal Trial; and his institution, the Icahn School of Medicine at Mount Sinai, receives royalty payments from Edwards Lifesciences and Medtronic for intellectual property related to Dr. Adams' involvement in the development of valve repair rings. The authors have no other conflicts of interest to declare.

Ethical Statement: The authors are accountable for all aspects of the work in ensuring that questions related to the accuracy or integrity of any part of the work are appropriately investigated and resolved. All procedures performed in this study were in accordance with the Helsinki Declaration (as revised in 2013). The manuscript is waived from patient informed consent according to the ethics committee or institutional review board.

Open Access Statement: This is an Open Access article distributed in accordance with the Creative Commons Attribution-NonCommercial-NoDerivs 4.0 International License (CC BY-NC-ND 4.0), which permits the noncommercial replication and distribution of the article with the strict proviso that no changes or edits are made and the original work is properly cited (including links to both the formal publication through the relevant DOI and the license). See: https://creativecommons.org/licenses/by-nc-nd/4.0/.

\section{References}

1. Nkomo VT, Gardin JM, Skelton TN, et al. Burden of

doi: 10.21037/jovs-2019-12

Cite this article as: Rimsukcharoenchai C, Pandis D, El-

Eshmawi A, Anyanwu AC, Adams DH. Complex mitral valve regurgitation: surgical evaluation, approach and repair techniques. J Vis Surg 2021;7:15. valvular heart diseases: a population-based study. Lancet 2006;368:1005-11.

2. Adams DH, Anyanwu AC. Seeking a Higher Standard for Degenerative Mitral Valve Repair: Begin with Etiology. J Thorac Cardiovasc Surg 2008;136:551-6.

3. Castillo JG, Anyanwu AC, Fuster V, et al. A near 100\% repair rate for mitral valve prolapse is achievable in a reference center: Implications for future guidelines. J Thorac Cardiovasc Surg 2012;144:308-12.

4. Castillo JG, Anyanwu AC, El-Eshmawi A, et al. All anterior and bileaflet mitral valve prolapses are repairable in the modern era of reconstructive surgery. Eur J Cardiothorac Surg 2014;45:139-45.

5. Carpentier A, Adams DH, Filsoufi F. Carpentier's Reconstructive Valve Surgery: From Valve Analysis to Valve Reconstruction. 1st ed. Philadelphia: Saunders/ Elsevier, 2010.

6. Doherty JU, Kort S, Mehran R, et al. ACC/AATS/ AHA/ASE/ASNC/HRS/SCAI/SCCT/SCMR/STS 2017 Appropriate Use Criteria for Multimodality Imaging in Valvular Heart Disease: A Report of the American College of Cardiology Appropriate Use Criteria Task Force, American Association for Thoracic Surgery. J Am Coll Cardiol 2017;70:1647-72.

7. El-Eshmawi A, Alexis SL, Sengupta A, et al. Surgical management of mitral annular calcification. Curr Opin Cardiol 2020;35:107-15.

8. Varghese R, Anyanwu AC, Itagaki S, et al. Management of systolic anterior motion after mitral valve repair: An algorithm. J Thorac Cardiovasc Surg 2012;143:S2-7.

9. El-Eshmawi A, Anyanwu A, Boateng P, et al. Second Cross-Clamp to Perfect Degenerative Mitral Valve Repair - Decision Making Algorithm, Safety and Outcomes. J Thorac Cardiovasc Surg 2019;S00225223(19)32196-8. 